\title{
EDITORIAL
}

\section{Sobre nuestra revista: un número extraordinario en materia de Derecho del Trabajo y de la Seguridad Social}

\author{
LA Comisión GeStora DE IGUALDAD DE LA AEDTSS \\ Eva MaRÍa Blázquez Agudo
}

Directora de Femeris. Universidad Carlos III de Madrid orcid id: 0000-0002-8214-1960

doi: https://doi.org/10.20318/femeris.2019.4563

El primer número de nuestra revista se publicó en verano de 2016. Hasta ahora se han editado puntualmente dos números por año: uno en enero y otro en julio. En este período la revista ha sido indexada en diversas bases de datos; ÍnDICEs-CSIC, catálogo LATINDEX, referenciada en Dialnet, incluida en ERIH PLUS y analizada en MIAR (Matriu d'Informació per a l'Avaluació de Revistes). Queremos agradecer la aportación de quienes han publicado sus trabajos, pero, también a quienes han realizado las evaluaciones y han configurado los distinto comités.

Ahora, se va a extender la edición de la revista a tres números al año. A partir de 2019 se va a publicar anualmente, además de los dos números generales, un número extra en materia de Derecho del Trabajo y de la Seguridad Social con perspectiva de género, aunque también se incluirá una sección donde se recogerán estudios sobre igualdad y discriminación en general. Con este fin se ha firmado un convenio con la Asociación Española de Derecho del Trabajo y de la Seguridad Social, presidida por la profesora Casas Baamonde.

La Asociación Española de Derecho del Trabajo y de la Seguridad Social está integrada por profesionales de muy diverso perfil que desarrollan su actividad en el ámbito del Derecho del Trabajo y de la Seguridad Social, procedentes tanto de la universidad, de la magistratura, del ejercicio profesional, de la gestión de los recursos humanos, así como de otros campos de actuación relacionados. Una de sus finalidades principales es difundir las actividades promovidas por sus socios, así como fomentar el intercambio de conocimientos y experiencias docentes e investigadoras en el ámbito universitario y profesional y el debate científico. 
En el pasado Congreso de la Asociación Española de Derecho del Trabajo y la Seguridad Social, celebrado en Santiago de Compostela el 31 mayo 2018, la Asociación decidió la creación de una Comisión de Igualdad, para lo cual aprobó su Reglamento de Funcionamiento, y designó a las siete personas que actuarían como Gestora hasta la elección de su primera composición ordinaria en el Congreso de Salamanca de 2019.

Las personas integrantes de dicha Gestora, reunidas el pasado 4 de septiembre en la sede de la Asociación en Madrid, se constituyeron y empezaron a funcionar como Comisión de Igualdad con las funciones atribuidas. En general, sus funciones son el desarrollo de actuaciones de promoción de los derechos a la igualdad y no discriminación en el ámbito de las relaciones laborales, dentro de los términos de la legislación vigente, con una actuación que queremos sea lo más intensa posible en los ámbitos académico, formativo, informativo y divulgativo.

Dentro de este marco, las principales líneas de actuación son la puesta en marcha de una revista sobre igualdad en el ámbito de las relaciones laborales; un boletín periódico de noticias sobre igualdad; las bases para la elaboración de una base documental de acceso abierto sobre legislación, jurisprudencia y doctrina científica; el diseño de dos jornadas anuales sobre igualdad, una residenciada el 8 de marzo sobre una temática de igualdad de género, y otra sobre otras igualdades cuya temática y fecha se decidirán anualmente; la celebración de convenios con otras instituciones para la puesta en marcha de actividades conjuntas en cuestiones de igualdad; $y$, para canalizar y publicitar todas estas actividades, una pestaña de fácil acceso dentro de la página web de la Asociación.

Entre las actividades a desarrollar, como se ha señalado, está la de organizar una revista en materia de igualdad, y en este contexto, se decidió la firma el acuerdo con FEMERIS. Revista Multidisciplinar de Estudios de Género para desarrollar este número especial en materia de Derecho del Trabajo y de la Seguridad Social.

La organización de este número extraordinario será la misma que la de los números ordinarios: artículos, misceláneas, reseñas y varia. En las dos primeras secciones se exige la revisión por pares de los trabajos antes de la decisión sobre su publicación. En reseñas se incluirán recensiones en la materia y en varia se acogerán resumes de eventos en igualdad con el fin de dar a conocer las principales conclusiones de congresos, jornadas y seminarios. Pero, además, dado que la Comisión trata de abarcar actividades en todos los aspectos de la igualdad, se abrirá una nueva sección donde se publicarán trabajos en otros ámbitos distintos a los de la perspectiva de género, como puede ser la discapacidad, la edad, la etnia, entre otros.

Esperamos que el número especial en materia de Derecho del Trabajo y de la Seguridad Social aporte grandes colaboraciones en este ámbito, como hasta ahora ha ocurrido en los números generalistas. Así que seguid enviándonos vuestras aportaciones. 